\title{
Flache Erfahrungskurvenprofile von ausländischen Beschäftigten in Deutschland
}

\author{
Dieser Beitrag widmet sich der Entlohnungsstruktur von ausländischen und deutschen \\ sozialversicherungspflichtig Vollzeitbeschäftigten und zielt auf die Lohnzuwächse durch \\ die Aneignung von zusätzlicher Erfahrung auf dem deutschen Arbeitsmarkt. Bisherige \\ Erkenntnisse für Deutschland deuten darauf hin, dass Arbeitsmarkterfahrung bei Ausländern \\ im Vergleich zu deutschen Beschäftigten zu keinen oder nur geringen Lohnzuwächsen \\ führt. Wir identifizieren und diskutieren Ursachen und Erklärungen für diese flacheren \\ Erfahrungskurvenprofile, was insbesondere vor dem Hintergrund der Einkommensungleichheit \\ zwischen beiden Gruppen relevant ist.
}

\begin{abstract}
Spätestens seit den Immigrationswellen ab 2015 stehen ausländische Beschäftigte im Fokus der deutschen Arbeitsmarktforschung. Ein oft aufgegriffener Aspekt der Arbeitsmarktforschung ist das Lohngefälle zwischen einheimischen und ausländischen Beschäftigten. Damit gehen unweigerlich die Fragen einher, inwieweit Personen mit Migrationshintergrund geringere Verdienste aufweisen, worauf die Unterschiede zurückgeführt werden können und ob es zu einer Konvergenz der Löhne kommt. Diese Frage ist Gegenstand jahrzehntelanger Diskussionen in der Literatur. Eine Antwort hängt von einer Reihe unterschiedlicher Faktoren wie der gewählten AnalyseMethodik und den verwendeten Daten ab. Aber nicht nur methodische Gründe spielen eine Rolle, so können Unterschiede in der Entlohnung durch Sprachbarrieren, nicht vorhandene oder nicht anerkannte Berufsabschlüsse, fehlende Arbeitsmarkterfahrung, aber auch systematische Benachteiligung in Form von Diskriminierung resultieren. Entsprechend sind für eine gelungene Integration von Personen aus dem Ausland wissenschaftlich begründete Erkenntnisse von zentraler Bedeutung, da sie die Basis für politische Handlungsempfehlungen bilden.
\end{abstract}

In diesem Aufsatz fokussieren wir uns auf die Erklärungen flacher Erfahrungskurven bei ausländischen Arbeitnehmern in Deutschland, wie sie immer wieder in der Literatur gefunden, jedoch nie im Detail analysiert und erklärt wurden. Für Deutschland liegen diverse Untersuchungen vor, die zeigen, dass ausländische Arbeitnehmer im Durch-

(c) Der/die Autor:in 2021. Open Access: Dieser Artikel wird unter der Creative Commons Namensnennung 4.0 International Lizenz veröffentlicht (creativecommons.org/licenses/by/4.0/deed.de).

Open Access wird durch die ZBW - Leibniz-Informationszentrum Wirtschaft gefördert. schnitt deutlich niedrigere Löhne erwirtschaften. Zum anderen zeigt die bisherige Literatur, dass es nur bedingt $z u$ einer Lohnkonvergenz zwischen ausländischen und deutschen Beschäftigten kommt. Die Studien (z. B. Licht und Steiner, 1994; Schmidt, 1997; oder Zibrowius, 2012) legen nahe, dass ausländische Beschäftigte in Deutschland flachere Erfahrungskurvenprofile aufweisen und damit weniger von ihrer erworbenen Arbeitsmarkterfahrung profitieren. Demnach schöpfen Personen aus dem Ausland im Vergleich zu Einheimischen geringere Lohnzuwächse aus jedem zusätzlichen Jahr an Arbeitsmarkterfahrung in Deutschland. Eine aktuellere Studie von Lehmer und Ludsteck (2015) kommt zwar zu dem Ergebnis, dass sich das Lohngefälle zwischen ausländischen und deutschen Arbeitnehmern innerhalb der ersten acht Jahre um 14 Prozentpunkte schließt. Das wird jedoch hauptsächlich auf Veränderungen in den beobachteten Charakteristiken, wie beispielsweise Berufs- und Firmenstruktur, zurückgeführt. Somit finden die Autoren zwar eine Lohnkonver-

Prof. Dr. Stephan Brunow ist Professor für Volkswirtschaftslehre und Arbeitsmarktökonomik an der Hochschule der Bundesagentur für Arbeit $(\mathrm{HdBA})$ in Schwerin.

Oskar Jost ist wissenschaftlicher Mitarbeiter am Institut für Arbeitsmarkt- und Berufsforschung (IAB), Forschungsbereich Regionales Forschungsnetz, in Berlin. 
genz der beiden Gruppen, jedoch bleibt der Aspekt der vergleichsweise niedrigeren Entlohnung der (potenziell gleichen) Arbeitsmarkterfahrung unberücksichtigt.

Vor dem Hintergrund dieser Studienergebnisse stellt sich die Frage, ob und welche Faktoren die bestehenden Unterschiede in den Erfahrungskurvenprofilen zwischen den Gruppen erklären und nivellieren können. Um Antworten auf diese Fragen zu finden, bedarf es einer empirischen Analyse, in der alternative Erklärungen und Einflüsse kontrolliert werden müssen. Ferner sind Daten mit einem langen Beobachtungshorizont nötig, um etwaige Veränderungen, Arbeitslosigkeitsphasen, Karrierewege und Lohnkonvergenzen im Laufe der Erwerbsbiogragrafien zu erfassen. Wir greifen hierfür auf die vollständigen Erwerbsbiografien des Instituts für Arbeitsmarkt- und Berufsforschung (IAB) zurück und wenden eine Oaxaca-Blinder-Lohnzerlegung an. Dabei richten wir unser Augenmerk auf die Erfahrungskurvenprofile der betrachteten Gruppen. Die kontrafaktische Analyse ermöglicht einen Vergleich der jeweiligen Einflüsse, sodass zum einen etwaige Unterschiede deutlich werden. Zum anderen können wir mithilfe der verwendeten Mikrodaten Antworten auf die Fragen finden, welche Faktoren zur Angleichung der Erfahrungskurven beitragen. Nicht zuletzt aus dem Blickwinkel der Debatte eines möglichen zukünftigen Fachkräftemangels fokussieren wir uns auf einen arbeitsmarktnahen Personenkreis mit anerkannten Berufsabschlüssen bzw. einem Hochschulabschluss in Vollzeitbeschäftigung.

Unsere Analyse zeigt, dass nach 15 Jahren auf dem deutschen Arbeitsmarkt jegliche Unterschiede in den Erfahrungskurven zwischen den Gruppen verschwinden. Dies deutet auf einen Konvergenzprozess hin, bei dem ausländische Beschäftigte lange Zeit benötigen, um im Vergleich zu Einheimischen identische Zugewinne aus ihrer Arbeitsmarkterfahrung zu generieren. Weiterhin können wir Unterschiede in den Erfahrungskurven ausschließlich für jüngere Beschäftigte bis 34 Jahren identifizieren. Damit sind in erster Linie junge Arbeitnehmer durch diese Defizite betroffen, wohingegen ältere Ausländer keine Unterschiede zur deutschen Belegschaft aufweisen. Anerkannte Bildungsabschlüsse bzw. eine Ausbildung in Deutschland, aber auch die Annahme der deutschen Staatsbürgerschaft, sind mit einer Annäherung in der Entlohnung verbunden.

\section{Daten, Identifikation und Methodik}

Für unsere Analyse verwenden wir einen umfangreichen Auszug der Integrierten Erwerbsbiografien (IEB) des IAB mit Informationen zu rund $10 \%$ aller Beschäftigten in Deutschland. Diese Daten enthalten unter anderem Infor- mationen zu Löhnen, einzelnen Beschäftigungsepisoden, Geschlecht, Wohn- und Arbeitsort oder Staatsangehörigkeit der abhängig Beschäftigten für die Jahre 1975 bis 2015. Da es sich ausschließlich um sozialversicherungspflichtig Beschäftigte handelt, betrachten wir keine Selbständigen und Beamte.

Mithilfe der zur Verfügung stehenden Erwerbsbiografien erstellen wir Variablen zur individuellen Arbeitsmarktnähe, die wir als Querschnitt für das Jahr 2015 aufbereiten. ${ }^{1}$ In der Analyse berücksichtigen wir ausschließlich Beschäftigte in Vollzeit, da unsere Daten keine Informationen zur Wochenarbeitszeit enthalten und Arbeit in Vollzeit besser vergleichbar ist. Weiterhin wird die Analyse ausschließlich für Beschäftigte durchgeführt, die über einen berufsqualifizierenden Abschluss wie eine Berufsausbildung oder ein Studium verfügen. Die Unterteilung zwischen deutschen und ausländischen Beschäftigten nehmen wir anhand der in den Daten gemeldeten Staatsangehörigkeit vor. Dabei werden alle Personen, die keine deutsche Staatsangehörigkeit zum Stichtag des 15. September 2015 aufweisen als Personen aus dem Ausland klassifiziert. Diejenigen, die im Zuge ihres Erwerbslebens ihre Staatsangehörigkeit zur deutschen Staatsangehörigkeit wechseln, werden in der Analyse ausgeschlossen. Diese Gruppe wird allerdings nochmals separat betrachtet.

Methodisch bauen wir unsere Untersuchung auf der Oaxaca-Blinder-Lohnzerlegung auf, die üblicherweise auch zur Analyse des Gender-Pay-Gaps verwendet wird. Mithilfe dieser Zerlegung können wir die Lohndifferenz zwischen den ausländischen und deutschen Beschäftigten in drei Teile zerlegen²: in einen Ausstattungseffekt, einen Koeffizienteneffekt und einen Interaktionseffekt. ${ }^{3}$ Der Ausstattungseffekt misst den Einfluss auf die Lohndifferenz, der aus Unterschieden in den beobachteten Merkmalen resultiert wie z.B. der unterschiedlichen Arbeitsmarkterfahrung (in Jahren gemessen) zwischen den beiden Gruppen. Der Koeffizienteneffekt, der für unsere Analyse von zentraler Bedeutung ist, misst hingegen den Effekt auf die Lohndifferenz, der z. B. aus Unterschieden in der Vergütung eines zusätzlichen Jahres Arbeitsmarkterfahrung resultiert (Preis- oder Steigungseffekt). ${ }^{4}$ Der Interaktionsterm stellt eine Multiplikation der beiden vorherigen Effekte dar und kann für unsere Analyse vernach-

1 Für mehr Informationen zur Aufbereitung siehe Brunow und Jost (2021).

2 Damit weicht diese Lohnzerlegung etwas von der häufig verwendeten zweiteiligen Lohnzerlegung nach Oaxaca-Blinder ab, siehe hierzu Jones und Kelley (1984).

3 Siehe zur Methodik auch Schuler (2008).

4 Um Unterschiede in den Koeffizienten zwischen beiden Gruppen zu identifizieren, wird die durchschnittliche Arbeitsmarkterfahrung in Jahren der deutschen Arbeitnehmer als Referenz herangezogen. 
Tabelle 1

Ergebnisse der Lohnzerlegung

\begin{tabular}{lcc} 
& $\begin{array}{c}\text { Zerlegung } \\
\text { des Lohn- } \\
\text { gefälles }\end{array}$ & Differenz in \% \\
\hline Lohn Ausländer / Deutsche & 0,779 & $-22,10$ \\
\hline Ausstattungseffekt & $\begin{array}{c}0,790^{\star \star \star} \\
(-0,003)\end{array}$ & $-21,00$ \\
\hline Koeffizientenffekt & $\begin{array}{c}0,982^{* \star *} \\
(-0,002)\end{array}$ & $-1,80$ \\
\hline Interaktion & $0,004^{\star}$ & 0,40 \\
\hline N Personen gesamt & $(-0,002)$ & \\
\hline N Ausländer & 1.344 .478 & \\
\hline N Deutsche & 96.334 & \\
\hline
\end{tabular}

Anmerkungen: Es sind ausschließlich Beschäftigte in Vollzeit und mit einem berufsqualifizierenden Abschluss berücksichtigt, die eine Information zum Bildungsabschluss aufweisen. ${ }^{*} 10 \%,{ }^{*} 5 \%$, ${ }^{* *} 1 \%$, Standardfehler sind auf Firmenebene geclustert und in Klammern.

Quelle: eigene Berechnungen.

lässigt werden, da hierfür kein nennenswerter Einfluss auf die Lohndifferenz vorliegt.

\section{Ergebnisse der empirischen Analyse}

Für unsere Analyse setzen wir auf eine Mincer-Lohngleichung, die wir vorher nach gängigen Kriterien kalibrieren, um eine robuste Modellspezifikation für die Lohnzerlegung nutzen zu können. Die Ergebnisse für die Lohnzerlegung sind dabei nachfolgend in Tabelle 1 dargestellt.

Grundsätzlich zeigt sich ein Lohngefälle zwischen den beiden Gruppen in Höhe von 22,1\% zugunsten der deutschen Beschäftigten. Eine kontrafaktische Angleichung der beobachteten Merkmale (Ausstattungseffekt) zwischen den Gruppen würde zu einer Lohnreduktion von $21 \%$ für deutsche Beschäftigte führen. Demnach hätten deutsche Beschäftigte nahezu das gleiche Lohnniveau wie ausländische Beschäftigte, wenn sie eine identische Verteilung in den beobachteten Merkmalen aufweisen würden. Der Koeffizienteneffekt zeigt, dass eine Angleichung der Koeffizienten zwischen den Gruppen zu einer Lohnreduktion in Höhe von 1,8\% für deutsche Beschäftigte zur Folge hätte.

Diese Effekte werden nachfolgend in Variablengruppen dargestellt, sodass eine detailliertere Betrachtung der Bestimmungsfaktoren der Lohndifferenz möglich ist, um insbesondere etwaige Unterschiede in den Erfahrungskurvenprofilen zu identifizieren. Die Interpretation erfolgt dabei analog zur vorher dargestellten Übersicht.
Tabelle 2

Ausstattungs- und Koeffizienteneffekt der Lohnzerlegung

\begin{tabular}{|c|c|c|c|c|}
\hline & \multicolumn{2}{|c|}{ Ausstattungseffekt } & \multicolumn{2}{|c|}{ Koeffizienteneffekt } \\
\hline & (1) & in $\%$ & (2) & in $\%$ \\
\hline Räumliche Verteilung & $\begin{array}{l}1,014^{\star * \star} \\
(-0,001)\end{array}$ & $+1,40$ & $\begin{array}{l}0,981^{* * *} \\
(-0,003)\end{array}$ & $-1,90$ \\
\hline Industrie & $\begin{array}{l}0,972^{\star * *} \\
(-0,001)\end{array}$ & $-2,80$ & $\begin{array}{c}0,998 \\
(-0,004)\end{array}$ & $-0,20$ \\
\hline Beruf & $\begin{array}{l}0,973^{* * *} \\
(-0,001)\end{array}$ & $-2,70$ & $\begin{array}{l}1,010^{* * *} \\
(-0,003)\end{array}$ & $+1,00$ \\
\hline Anforderungsniveau & $\begin{array}{l}0,974^{\star \star \star} \\
(-0,001)\end{array}$ & $-2,60$ & $\begin{array}{r}0,982^{* *} \\
(-0,007)\end{array}$ & $-1,80$ \\
\hline $\begin{array}{l}\text { Individuenspezifische } \\
\text { Merkmale }\end{array}$ & $\begin{array}{l}0,998^{* * *} \\
(0,000)\end{array}$ & $-0,20$ & $\begin{array}{l}0,997^{* * *} \\
(-0,001)\end{array}$ & $-0,30$ \\
\hline Bildung & $\begin{array}{l}1,005^{\star \star \star} \\
(-0,001)\end{array}$ & $+0,50$ & $\begin{array}{l}1,019^{\star \star *} \\
(-0,004)\end{array}$ & $+1,90$ \\
\hline Arbeitsmarkterfahrung & $\begin{array}{c}0,900^{\star * *} \\
(-0,002) \\
\end{array}$ & $-10,00$ & $\begin{array}{l}0,944^{* * *} \\
(-0,003) \\
\end{array}$ & $-5,60$ \\
\hline Firmencharakteristika & $\begin{array}{l}0,935^{\star \star \star} \\
(-0,001)\end{array}$ & $-6,50$ & $\begin{array}{c}1,111^{\star * *} \\
(-0,015)\end{array}$ & $+11,10$ \\
\hline Konstante & & & $0,950^{\star * *}$ & $-5,00$ \\
\hline
\end{tabular}

Anmerkungen: robuste Standardfehler in Klammern; * $10 \%$, ** $5 \%$, *** $1 \%$.

Quelle: eigene Berechnungen.

In Tabelle 2 ist in Spalte (1) der Ausstattungseffekt dargestellt, sodass die einzelnen Einflüsse der Zusammensetzung der beobachteten Lohndifferenz sichtbar werden. Hier zeigt sich, dass Unterschiede in der beobachteten Arbeitsmarkterfahrung von zentraler Bedeutung bei der Erklärung des Lohngefälles darstellen. Hätten demnach deutsche Beschäftigte die durchschnittliche Arbeitsmarkterfahrung wie ausländische Arbeitnehmer, würde der Lohn für deutsche Beschäftigte um $10 \%$ niedriger ausfallen. Damit gibt es hier deutliche Ausstattungsunterschiede zwischen den Gruppen. Die durchschnittlich niedrigere Arbeitsmarkterfahrung hängt dabei unter anderem mit einem durchschnittlich jüngeren Alter der ausländischen Beschäftigten zusammen. Um jedoch flachere Erfahrungskurvenprofile identifizieren zu können, müssen Unterschiede in den Koeffizienten aufgegriffen werden.

Die detaillierte Betrachtung des Koeffizienteneffektes in Spalte (2) von Tabelle 2 zeigt, wie auch bei der Ausstattung zuvor, deutliche Unterschiede in der Bewertung der Arbeitsmarkterfahrung. Demnach fällt die Vergütung der Arbeitsmarkterfahrung bei ausländischen Beschäftigten tatsächlich niedriger aus, was auf flachere Erfahrungskurvenprofile hindeutet. Würden deutsche Beschäftigte so wie die ausländischen Beschäftigten für ihre Arbeits- 
Tabelle 3

Unterschiede in den Erfahrungskurven nach Jahren in Beschäftigung

\begin{tabular}{lccc} 
Arbeitsmarkterfahrung & $<10$ Jahre & 10 bis 15 Jahre & $>15$ Jahre \\
\hline Effekt & $\begin{array}{c}0,940^{\star * *} \\
(-0,004)\end{array}$ & $\begin{array}{c}0,971^{\star * *} \\
(-0,004)\end{array}$ & $\begin{array}{c}0,993 \\
(-0,006)\end{array}$ \\
\hline in \% & $-6,00$ & $-2,90$ & $-0,70$ \\
\hline
\end{tabular}

Anmerkungen: robuste Standardfehler in Klammern; * $10 \%,{ }^{\star \star} 5 \%$, ${ }^{\star \star \star} 1 \%$. Quelle: eigene Berechnungen.

markterfahrungen entlohnt werden, würden sie 5,6 \% weniger verdienen.

Damit bestätigen sich die Ergebnisse der anfangs genannten Untersuchungen für den deutschen Raum. Die flacheren Profile führen dazu, dass zusätzlich erworbene Arbeitsmarkterfahrung ausländischer Arbeitnehmer weniger stark vergütet wird als die der deutschen Beschäftigten, sodass dies einer Lohnkonvergenz entgegenwirkt.

Die bisher vorliegende Literatur zu diesem Thema endet an dieser Stelle und gibt keine klare Antworten auf die Frage, welche Faktoren die bestehenden Unterschiede in den Erfahrungskurvenprofilen möglicherweise nivellieren könnten und somit zu einer Konvergenz der Löhne beitragen würden. Denn genau diese Gründe sind unabdingbar für eine gelungene Integration ausländischer Beschäftigter auf dem Arbeitsmarkt. Mithilfe der von uns verwendeten Daten identifizieren wir einige Faktoren, die zu einer Konvergenz der Erfahrungskurven beitragen und stellen sie nachfolgend vor.

\section{Vorhandensein eines Berufsabschlusses}

Unsere Ergebnisse betrachten Personen, die eine Berufsausbildung nachweisen konnten. Erweitern wir den Personenkreis um jene Beschäftigte ohne Berufsausbildung - bei denen ausländische Personen überrepräsentiert sind - fallen die Erfahrungsprofile aller ausländischen Beschäftigten flacher aus. Statistisch wirkt sich dies allerdings auch auf die anderen ausländischen Beschäftigten mit anerkannten Berufsabschluss aus. Damit erklärt die (Nicht-) Anerkennung von ausländischen Berufsabschlüssen teilweise die flacheren Erfahrungsprofile. Betrachtet man ausschließlich Personen mit einem Lehrabschluss und schließt Personen mit einem Studienabschluss aus, nähern sich bereits die Erfahrungsprofile an. Weiterhin zeigt sich, dass ausländische Beschäftigte, die ihre Berufsausbildung in Deutschland absolvierten, keine signifikanten Unterschiede in den Erfahrungsprofilen gegenüber den Einheimischen aufweisen.
Tabelle 4

Unterschiede in den Erfahrungsgruppen nach Altersgruppen

\begin{tabular}{lccccc} 
Altersgruppen & $16-24$ & $25-34$ & $35-44$ & $45-54$ & $55-64$ \\
\hline Arbeitsmarkterfahrung & $\begin{array}{c}0,962^{* \star *} \\
(0,009)\end{array}$ & $\begin{array}{c}0,958^{\star * *} \\
(0,005)\end{array}$ & $\begin{array}{c}0,990 \\
(0,006)\end{array}$ & $\begin{array}{c}0,981^{*} \\
(0,010)\end{array}$ & $\begin{array}{c}0,972 \\
(0,019)\end{array}$ \\
\hline in \% & $-3,80$ & $-4,20$ & $-1,00$ & $-1,90$ & $-2,80$ \\
\hline
\end{tabular}

Anmerkungen: robuste Standardfehler in Klammern; * $10 \%,{ }^{* \star} 5 \%,{ }^{* \star *} 1 \%$. Quelle: eigene Berechnungen.

\section{Angleichung der Erfahrungskurvenprofile}

Der wichtigste Faktor, der zur Angleichung der Erfahrungskurven zwischen den Gruppen führt, ist eine längerfristige Beschäftigung auf dem deutschen Arbeitsmarkt. So zeigt sich, dass ausländische Beschäftigte, die mindestens 15 Jahre auf dem deutschen Arbeitsmarkt aktiv sind, keine signifikanten Unterschiede in den Erfahrungskurvenprofilen aufweisen, wie in Tabelle 3 dargestellt.

Das Phänomen der unterschiedlichen Erfahrungskurven nimmt hierbei sukzessive über die Jahre ab. Dies kann auf eine Reihe unterschiedlicher Einflüsse zurückgeführt werden, wie eine Verbesserung der Sprachkenntnisse ausländischer Arbeitnehmer oder eine verbesserte Marktübersicht, die mit anderen Karrierepfaden verbunden ist.

Ferner zeigt unsere Analyse, dass flache Erfahrungskurven auf bestimmte Altersgruppen beschränkt sind. Demnach weisen besonders junge Personen aus dem Ausland Defizite auf, wie in Tabelle $4 \mathrm{zu}$ sehen ist. Diese Unterschiede nehmen dabei ab einem Alter von 35 Jahren ab bzw. sind nicht mehr signifikant.

Ähnlich interessante Ergebnisse zeigen sich, wenn wir ein Matchingverfahren verwenden, bei dem deutsche und ausländische Beschäftigte nach bestimmten Kriterien einander zugeordnet werden. ${ }^{5}$ Für die Auswahl von sogenannten statistischen Zwillingen nutzen wir hierfür folgende Kriterien: Geschlecht, Altersgruppe, Bildungsabschluss, bisherige Arbeitsmarkterfahrung und Arbeitsmarktregion. Die Ergebnisse sind in Tabelle 5 in der ersten Zeile abgetragen. Bei der Betrachtung statistischer Zwillinge zeigen sich keine Unterschiede in den Erfahrungskurven zwischen den Gruppen.

Ferner kann die Annahme der deutschen Staatsbürgerschaft als starker Indikator für den individuellen Integra-

5 Wir nutzen hierfür das CEM-Matchingverfahren nach Blackwell et al. (2009). 
Tabelle 5

Unterschiede in den Erfahrungsprofilen

\begin{tabular}{|c|c|c|c|}
\hline & & $\begin{array}{r}\text { Koeffizi } \\
\text { für die Arbeit }\end{array}$ & $\begin{array}{l}\text { fekt } \\
\text { erfahrung }\end{array}$ \\
\hline & & Schätzwert & in $\%$ \\
\hline (1) & Matchingverfahren & $\begin{array}{c}0,998 \\
(-0,004)\end{array}$ & 0,20 \\
\hline (2) & DE-Staatsbürgerschaft & $\begin{array}{l}1,025^{\star \star *} \\
(-0,002)\end{array}$ & 2,50 \\
\hline (3) & $\begin{array}{l}\text { Eingebürgerte und Deut- } \\
\text { sche }\end{array}$ & $\begin{array}{l}0,983^{\star \star \star} \\
(0,004)\end{array}$ & $-1,70$ \\
\hline
\end{tabular}

Anmerkungen: robuste Standardfehler in Klammern; ${ }^{*} 10 \%,{ }^{* \star} 5 \%,{ }^{* \star \star} 1 \%$. Quelle: eigene Berechnungen.

tionswillen aufgefasst werden. Ausländische Personen, welche die deutsche Staatsbürgerschaft annahmen, haben steilere Erfahrungsprofile relativ zu denen, die derzeit als ausländische Beschäftigte in Deutschland tätig sind. Die Löhne steigen um 2,5\% (vgl. Tabelle 5, Zeile 2). Ein Vergleich der eingebürgerten Ausländer mit deutschen Beschäftigten (Zeile 3) weist auf eine gewisse Konvergenz hin, der Unterschied beträgt nun nur noch 1,7\%.

\section{Fazit und Implikation}

Zunächst hat die Analyse Erkenntnisse aus der Literatur bestätigt, wonach ausländische Beschäftigte in Deutschland tatsächlich deutlich flachere Erfahrungskurvenprofile aufweisen als deutsche Beschäftigte. Dies führt in erster Linie dazu, dass zusätzlich erworbene Arbeitsmarkterfahrung weniger stark honoriert wird als die der deutschen Beschäftigten, was einer Konvergenz der Lohnniveaus entgegensteht.

Mithilfe der zugrunde gelegten, repräsentativen Daten konnten wir einige Faktoren aufzeigen, die zu einer Angleichung der Erfahrungsprofile beitragen. So zeigt sich, dass jegliche messbaren Unterschiede nach 15 Jahren verschwinden und ausländische Beschäftigte identische Zugewinne aus zusätzlicher Arbeitsmarkterfahrung schöpfen können. Weiterhin wird deutlich, dass flachere Erfahrungskurven auf Jüngere beschränkt sind und für ältere Beschäftigte nicht mehr auftreten. Die Anerkennung von ausländischen Ausbildungsabschlüssen oder eine Ausbildung in Deutschland sind auch hervorzuheben, wie die Annahme der deutschen Staatsbürgerschaft als zentrale Hebel hin zu einer gleichen Entlohnung. Diese Ergebnisse bestätigen sich, wenn Ausländer und Deutsche mithilfe eines Matchingverfahres einander als statistische Zwillinge zugeordnet werden, welches alle Unterschiede in den Erfahrungskurven nivelliert. Damit kommen wir zum Schluss, dass in der Vergangenheit beobachtete flachere Erfahrungsprofile unter den Ausländern nicht auf mangelnde Integration oder einer generell unterschiedlichen Entlohnungsstruktur zurückzuführen sind. Insbesondere verschwinden sie mit der Aufenthaltsdauer in Deutschland, was ganz klar für den Abbau von Informationsasymmetrien spricht.

\section{Literatur}

Blackwell, M., S. M. lacus, G. King und G. Porro (2009), cem: Coarsened exact matching in Stata, The Stata Journal, 9 (4), 524-546.

Brunow, S. und O. Jost (2021, im Erscheinen): Wages of migrant and native employees in Germany: new light on an old issue, International Migration Review, online first.

Jones, F. L. und J. Kelley (1984), Decomposing Differences between Groups. A Cautionary Note on Measuring Discrimination, Sociological Methods \& Research, 12(3), 323-343.

Lehmer, F. und J. Ludsteck (2015), Wage assimilation of foreigners * which factors close the gap? Evidence from Germany, Review of In come and Wealth, 61(4), 677-701.

Licht, G. und V. Steiner (1994), Assimilation, labour market experience and earnings profiles of temporary and permanent immigrant workers in Germany, International Review of Applied Economics, 8(2), 130-156.

Schmidt, C. M. (1997), Immigrant Performance in Germany: Labor Earnings of Ethnic German Migrants and Foreign Guest-Workers, The Quarterly Review of Economics and Finance, 37, 379-397.

Schuler, B. (2008), Einkommensunterschiede zwischen Männern und Frauen, Wirtschaftsdienst, 88(8), 546-552, https://www.wirtschaftsdienst.eu/inhalt/jahr/2008/heft/8/beitrag/einkommensunterschiedezwischen-maennern-und-frauen.html (13. September 2021).

Zibrowius, M. (2012), Convergence or divergence? Immigrant wage assimilation patterns in Germany, SOEP papers, DIW Berlin, 479-2012.

Title: Flat Experience Curve Profiles of Foreign Employees in Germany

Abstract: This paper sheds light on the wage structure of foreign and German employees who are subject to social security contributions in fulltime employment and focuses on the experience structure. Existing evidence suggests that additional labour market experience is rewarded less for migrants than for their German counterparts. We identify and discuss reasons and explanations for migrants' flat experience curves, which is particularly important in the light of the debate on inequality between both groups. These explanations include: the recognition of vocational training degrees, the successful passing of vocational training in Germany, German citizenship by naturalisation, and the labour market experience. After ten to 15 years in the German labour market, we identify convergence in the returns to labour market experience.

JEL Classification: J15, J31, J44 